\author{
Bao-Yuan Guo ${ }^{1}$ \\ Songxue Wang ${ }^{2}$ \\ Baozhong Ren ${ }^{2}$ \\ Xin Li $\mathbf{i}^{1,3}$ \\ Fei Qin ${ }^{1,3}$ \\ Jianzhong $\mathrm{Li}^{1 *}$ \\ ${ }^{1}$ State Key Laboratory of \\ Environmental Chemistry and \\ Ecotoxicology Research Center \\ for Eco-Environmental Sciences, \\ Chinese Academy of Sciences, \\ Beijing, P. R. China \\ ${ }^{2}$ Academy of State \\ Administration of Grain, Beijing \\ P. R. China \\ ${ }^{3}$ The College of Art and Science \\ of Beijing Union University, \\ Beijing, P. R. China
}

Received October 9, 2009

Revised December 15, 2009

Accepted December 16, 2009

\section{Short Communication Citrinin selective molecularly imprinted polymers for SPE}

\begin{abstract}
Molecularly imprinted polymers (MIPs) for citrinin (Cit) with 1-hydroxy-2-naphthoic acid (HNA) as mimic template were prepared and the molecularly imprinted SPE method was developed for the detection of Cit in rice with HPLC. The adsorption properties of HNA and Cit on the MIPs and nonimprinted polymers were investigated. It proved that MIPS showed higher selectivity adsorption to HNA and Cit than nonimprinted polymers. The recoveries of $\mathrm{Cit}$ in rice were in the range of $86.7-97.7 \%$. The spiked rice samples and five rice samples in Beijing market were detected using molecularly imprinted SPE method and satisfactory results were obtained as discussed in this article.
\end{abstract}

\section{Keywords: Citrinin / Mimic template / Molecularly imprinted SPE / Rice DOI 10.1002/jssc.200900652}

\section{Introduction}

Citrinin (Cit), a mycotoxin produced by Penicillium citrinum, is a kind of compound with highly toxic, mutagenic, teratogenic and carcinogenic properties, and has been implicated as a causative agent in human hepatic and extrahepatic carcinogenesis $[1,2]$. It is often found in crops, vegetables and fruits. Rice is one of the most important food crops in China, mainly produced and stored in Northeast China and in South China. Mould and mycotoxin contaminations are highly risky. Cit is one of the most important mycotoxins threatening the Chinese health seriously. The analytical method of Cit was commonly employed using immunoaffinity columns (IACs) to cleanup the sample extracted from complex food matrix [3]. The IACs are of high selectivity with its specific advantage over the conventional SPE methods. However, the IAC-SEP has its inherent limitations. The IACs could tolerate no organic solvents, and should be operated and preserved under critical conditions, such as aqueous media, well-controlled $\mathrm{pH}$ and ionic strength.

Correspondence: Dr. Bao-Yuan Guo, State Key Laboratory of Environmental Chemistry and Ecotoxicology Research Center for Eco-Environmental Sciences, Chinese Academy Sciences, P. O. Box 2871, Beijing 100085, P. R. China

E-mail: guoby@rcees.ac.cn

Fax: +86-10-69752382

Abbreviations: Cit, citrinin; HNA, 1-hydroxy-2-naphthoic acid; IAC, immunoaffinity columns; MAA, methacrylic acid; MIP, molecularly imprinted polymer; NIP, nonimprinted polymers
To address these problems, mycotoxin-selective molecularly imprinted polymers (MIPs) have been considered as a potential choice. Its high selectivity, enrichment capability and chemical stability make them quite suitable for the material of SPE for purifying the analytes in matrix. A number of MIPs that use mycotoxins have been developed [4-9]. The MIPs-based SPEs have been developed and used in the sample per treatment during mycotoxin analysis such as ochratoxin A [4-8] and zearalenone [9].

It has been observed that the binding of the templates to the polymers is sometimes so strong that it is difficult to remove the last trace of templates when noncovalent methods are used to prepare the polymers [10]. To overcome this drawback, scientists have synthesized MIPs using the analogues of the target molecules as templates (the templateanalogue, dummy or mimic template approach) [10-14]. In this way, if the MIPs release mimic template, they would not interfere with the quantitative detection of the target analytes. The present developed MIP methods for ochratoxin A were mostly based on the mimic template method by using benzyl(1-benzyloxy)-2-naphthoate or other compounds with similar structure [4-8], and the MIPs have been used in the SPE and sensor investigations successfully. The MIP materials for zearalenone by using cyclododecyl 2,4-dihydroxybenzoate as mimic template was prepared and used in the determination of zearalenone in red wine as well [9].

However, the MIPs for Cit have not yet been developed as per our knowledge, and the corresponding MIPs based on the SPE method have not been developed as well. This study prepared a MIP for the mycotoxins of Cit by using 1-hydroxy-2-naphthoic acid (HNA) as the mimic template.

\footnotetext{
*Additional correspondence: Prof. Jianzhong Li, E-mail: look99@263.net
} 
In order to quantify the amounts of these toxins in rice, a simultaneous analytical method using an HPLC system with fluorimetry detection was developed in the present work.

\section{Materials and methods}

\subsection{Chemicals and materials}

Cit, HNA and ethylene glycol dimethacrylate were purchased from Sigma-Aldrich (Sigma-Aldrich, Steinheim, Germany). Methacrylic acid (MAA) was purchased from Alfa Aesar (Johnson Mathey, USA). 2,2'-Azobisisobutyronitrile from Shanghai No. 4 Reagent \& H.V. Chemical (Shanghai, P. R. China) and was recrystallized from ethanol. All the solvents were of chromatographic grade.

The individual stock solutions of Cit were prepared at a concentration of $1000 \mathrm{mg} / \mathrm{L}$ in methanol, and the standard solutions of lower concentration were prepared by the serial dilution of the stock solutions. All stock and standard solutions were stored at $4^{\circ} \mathrm{C}$ in a refrigerator and reprepared every month. The methanol of HPLC grade was purchased from Merck (Darmstadt, Germany); water was doubly distilled. All other reagents were of analytical grade. All the HPLC analysis was done with a Shimadzu 10A HPLC system (Shimadzu, Japan) equipped with UV-Vis and fluorescence detector.

The HPLC method for the detection of HNA was as follows: a Shimadzu $\mathrm{C}_{18}$ column $(150 \mathrm{~mm} \times 4.6 \mathrm{~mm}$, $5 \mu \mathrm{m})$ was used in the HPLC separation and thermostated at $30^{\circ} \mathrm{C}$. The mobile phase was methanol/water $(80: 20, \mathrm{v} / \mathrm{v})$, pHs of methanol and water were adjusted with $\mathrm{H}_{3} \mathrm{PO}_{4}$ to 4.1 and 4.6, respectively. The flow rate was $0.9 \mathrm{~mL} / \mathrm{min}$, the detection wavelength was $220 \mathrm{~nm}$ and injection volume was $10 \mu \mathrm{L}$.

The mobile phase for Cit was acetonitrile/water (1:1, $\mathrm{v} / \mathrm{v})$. The $\mathrm{pH}$ values of acetonitrile and water were adjusted with acetic acid to 2.66 and 2.48 , respectively. The flow rate was $1 \mathrm{~mL} / \mathrm{min}$, and the excitation wavelength was $331 \mathrm{~nm}$ and the emission was $500 \mathrm{~nm}$.

\subsection{Synthesis and characterization of MIP}

\subsubsection{Polymer preparation}

A measure of $0.100 \mathrm{~g}$ HNA (as mimic template) was dissolved in $4.8 \mathrm{~mL}$ acetone, then $0.162 \mathrm{~mL}$ MAA was added. Then it was put into the ultrasonic bath for $30 \mathrm{~min}$ to enhance the interaction between MAA and HNA. Then,

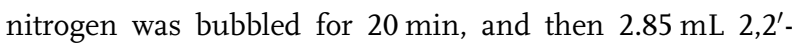
azobisisobutyronitrile initiator was added. The ampoule was sealed and the mixture was kept at $60^{\circ} \mathrm{C}$ in a water bath for $24 \mathrm{~h}$. The resulting hard polymers were crushed and ground to obtain regularly sized particles between 30 and $40 \mu \mathrm{m}$ suitable for the molecularly imprinted SPE evaluations.
Then they were extracted with methanol for 2 days using a Soxhlet extractor and washed with methanol-acetic acid 90:10 v/v until the template molecule could no longer be detected at $220 \mathrm{~nm}$ with a Shimadzu 2400 UV spectrophotometer (Shimadzu), and then washed with methanol to nature. Then the particles were vacuum dried at $50^{\circ} \mathrm{C}$. Control polymers (non-MIP, nonimprinted polymers, NIP) were prepared by the same procedure in the absence of template molecule.

\subsubsection{Static adsorption experiment}

The eleven equal parts of polymer particles $(20 \mathrm{mg})$ were mixed with $5 \mathrm{~mL}$ HNA or Cit solution with different concentration in a $10 \mathrm{~mL}$ conical centrifugation tube and sealed. The tubes were shaken in a water bath for at least $24 \mathrm{~h}$. Then the mixture was centrifuged for $10 \mathrm{~min}$ and Cit and HNA concentrations in the liquid phase were measured using HPLC. The amount of HNA and Cit bound to the polymer was calculated by subtracting the concentration of free HNA and Cit from the initial adsorbate loading.

\subsection{Preparation of rice samples}

All the rice samples were purchased from the local market, which were produced in Northeast China. A measure of $1.0 \mathrm{~g}$ ground rice sample $(<0.2 \mathrm{~mm})$ was ultrasonically extracted with $5 \mathrm{~mL}$ methanol/water $(7: 3, \mathrm{v} / \mathrm{v})$ for $10 \mathrm{~min}$, then centrifuged at $12000 \mathrm{rpm}$ for $10 \mathrm{~min}$. The solution was filtered with $0.45 \mu \mathrm{m}$ filter and directly loaded to the SPE.

\subsection{SPE}

\subsubsection{The SPE extraction of HNA}

The cartridges were prepared by packing $250 \mathrm{mg}$ of the polymer into empty $5 \mathrm{~mL}$ SPE cartridge with polyethylene first. Prior conditioning was carried out with $10 \mathrm{~mL}$ of methanol followed by $10 \mathrm{~mL}$ of water to wet the polymer completely. Then $5 \mathrm{~mL}$ of $0.05 \mathrm{mg} / \mathrm{L}$ HNA solution was uploaded to the SPE filled with MIP or NIP, then washed with $2 \mathrm{~mL}$ methanol, methanol/acetic acid (99:1, v/v) and methanol/acetic acid (98:2, v/v). The solution was blown with $\mathrm{N}_{2}$ to $1 \mathrm{~mL}$ and then analyzed with HPLC.<smiles>O=C(O)c1ccc2ccccc2c1O</smiles>

1-Hydroxy-2-naphthoic acid<smiles>CC1=C2C(=COC1C)C(O)=C(C(=O)O)C(=O)C2C</smiles>

Citrinin
Figure 1. The structures of HNA and Cit. 


\subsubsection{The SPE extraction of Cit}

A volume of $5 \mathrm{~mL}$ of $0.05 \mathrm{mg} / \mathrm{L}$ Cit solution was uploaded to the SPE filled with MIP or NIP, then washed with $2 \mathrm{~mL}$ methanol, methanol/acetate (99:1, v/v), methanol/acetate $(98: 2, v / v)$, and acetonitrile. The solution was blown with $\mathrm{N}_{2}$ to $1 \mathrm{~mL}$ for HPLC analysis.

\section{Results and discussion}

\subsection{MIP preparation and characterization}

It is advisable to select the mimic templates when synthesizing mycotoxin MIPs due to its high toxicity and expense. HNA, which is similar to Cit from the viewpoint of structure as shown in Fig. 1, was selected as the mimic template in this study. When HNA was washed off from the imprinted polymers, the imprinted caves for HNA were left for the selective adsorption of Cit in the samples. Porous structure of both MIPs and NIPs could be observed by SEM and the pore size was about $500 \mathrm{~nm}$, as shown in Fig. 2. In the exception of the pore, some points (marked P in the SEM photo in Fig. 2) were observed in MIPs, which have not been seen in the NIPs. The points might be formed during polymerization by HNA as the polymerizing core. The templates in the points could not be washed off with common methods, which caused the release of templates during sample per concentration. The SEM photograph proved that it was necessary to employ a mimic template during imprinted polymer preparation. To evaluate the properties of the selectivity of MIPs, the following investigation was done.

In order to estimate the selectivity of the MIPs to templates and Cit, the static adsorption performance of HNA and Cit was investigated. Figure 3 shows the static isothermal adsorption curve of HNA and Cit on the MIP and NIP. MIPs showed selective adsorption of HNA and Cit by comparing with NIPs, which indicated the mimic templates was successfully selected. The selectivity of MIPs was evaluated with the ratio of salutation adoption concentration of MIPs to that of NIPs. The selectivity to HNA was 4.78 , which is higher than that of Cit (1.57), which proved that the imprinted caves marched the HNA molecules better than Cit.

\subsection{MIP-based SPE investigation}

The prepared MIP materials were used for SPE column filling. The eluting conditions were different from HNA to Cit since they are of different chemical properties. The eluting solutions (methanol, methanol/acetic acid (99:1), methanol/acetic acid (98:2) $2 \mathrm{~mL}$ ) were investigated for HNA. As shown in Fig. 4, under the eluting conditions, the recovery of HNA was higher on MIP than that on NIP. The eluting solution of methanol/acetic acid (99:1) is of the highest efficiency with a recovery of $93.69 \%$. While optimizing the eluting volume, methanol/acetic acid (99:1) 1, 2, 3 and $4 \mathrm{~mL}$ were employed and $3 \mathrm{~mL}$ was the optimized eluting volume with a recovery of $96.4 \%$. Although the recovery was higher while using $4 \mathrm{~mL}$ eluting solution, it is not recommended for real sample cleanup considering that it is not helpful to remove the impurities in the sample matrix.

However, while optimizing the eluting conditions for Cit, the eluting solution with $3 \mathrm{~mL}$ methanol, methanol/ acetic acid (99:1) and methanol/acetic acid (98:2), the

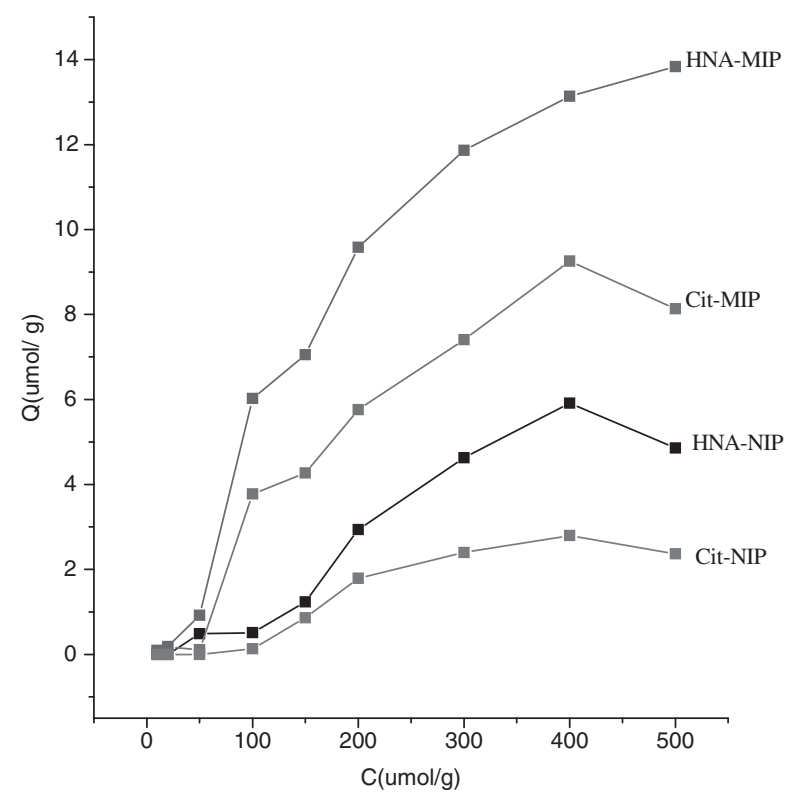

Figure 3. The isothermal adsorption curve of HNA and Cit on the MIP and NIP.

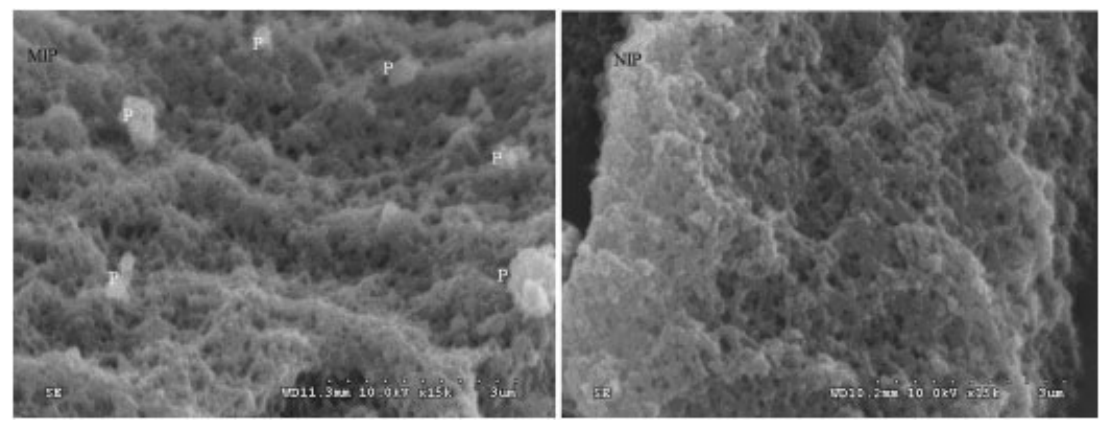

Figure 2. The SEM photograph of MIP and NIP at 15000 times. 
recoveries were not perfect and the highest recovery of $87.24 \%$ was obtained while using methanol/acetic acid (99:1) as shown in Fig. 4. While $3 \mathrm{~mL}$ acetonitrile was used as the eluting solution, the recovery of $97.17 \%$ was obtained, which is shown in Fig. 4. Therefore, the optimized eluting condition was employed with $3 \mathrm{~mL}$ acetonitrile.

\subsubsection{The rice sample investigation}

The detection limit of Cit by the present method was $0.5 \times 10^{-3} \mathrm{mg} / \mathrm{kg}$ and detection range of the method was from $1.5 \times 10^{-3}$ to $1 \mathrm{mg} / \mathrm{kg}$. The spiked rice samples were
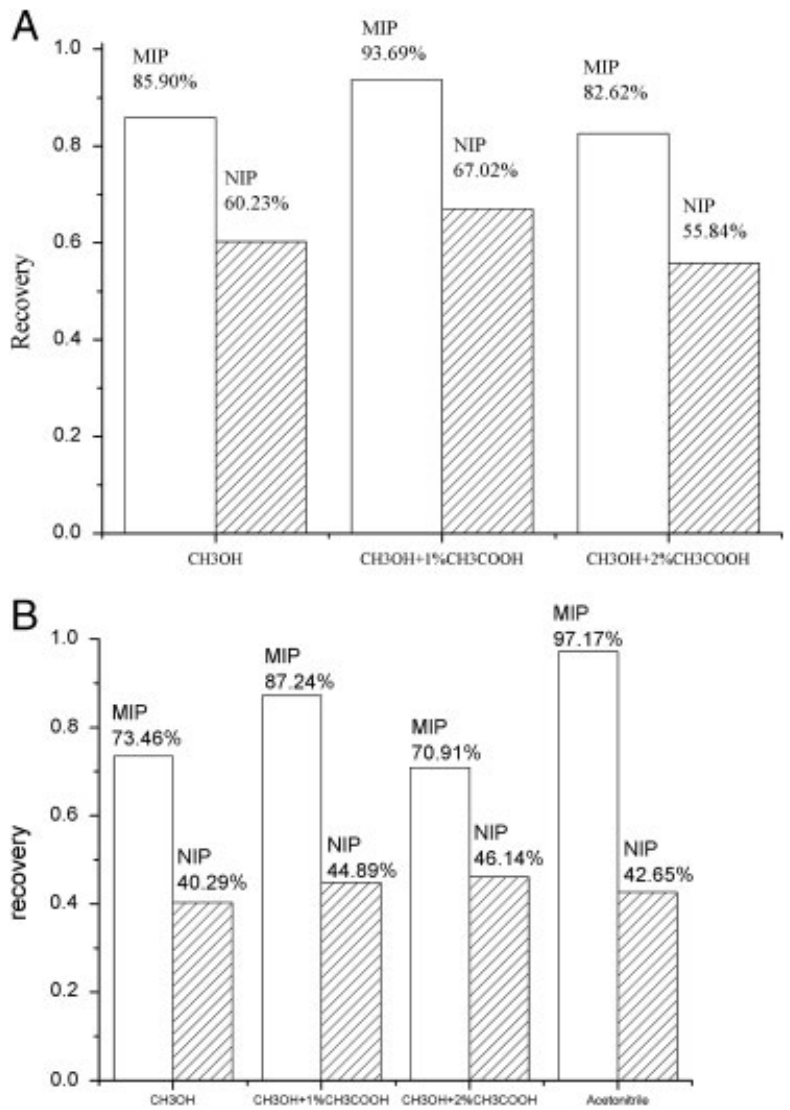

Figure 4. The elution condition for HNA (A) and elution condition for Cit (B). investigated in this study. Four concentrations of 0.005 , $0.01,0.05$ and $0.1 \mathrm{mg} / \mathrm{kg}$ were spiked in the rice, and the recoveries are summarized in Table 1 . Five rice samples obtained in the local market were analyzed Cit was lower than the detection limits in all the samples. The results proved that rice in the Beijing market was less polluted with Cit since the rice is mainly produced in Northeast China. The recoveries were in the range from 86.7 to $97.7 \%$. The RSD of the recovery was in the range from 1.2 to $3.8 \%$. The chromatogram of $0.05 \mathrm{mg} / \mathrm{L}$ Cit is shown in Fig. 5.

\section{Concluding remarks}

In this article, an MIP for Cit with HNA as mimic template was prepared successfully. The novel MIPs represented an excellent alternative to the affinity sorbents. The MIPs have both selective adsorption of the HNA and Cit. The MIPs showed high selectivity and yielded extractions with acceptable recovery for Cit. The method was used for determination of the spiked rice samples with rice and acceptable results were obtained.

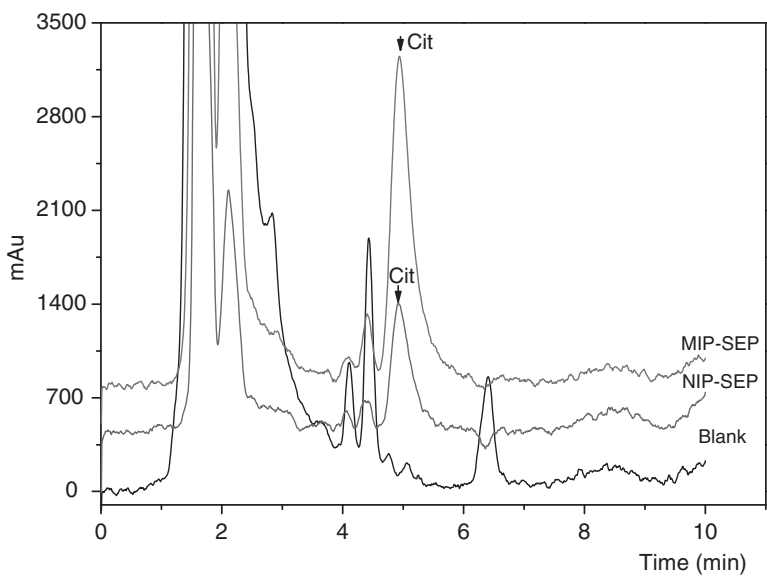

Figure 5. The chromatograms of spiked rice sample of 0.05 $\mathrm{mg} / \mathrm{L}$. No spike in the blank. The chromatogram of MIP-SPE is obtained by the pretreatment with MIP-based SPE and the chromatogram of NIP-SPE is obtained by the pretreatment with NIP-based SPE.

Table 1. The recoveries investigation of Cit in rice samples

\begin{tabular}{|c|c|c|c|c|c|c|c|c|}
\hline \multirow[t]{3}{*}{ Sample } & \multicolumn{8}{|c|}{ The addition concentration $(\mathrm{mg} / \mathrm{L})(n=3)$} \\
\hline & \multicolumn{2}{|c|}{0.005} & \multicolumn{2}{|c|}{0.01} & \multicolumn{2}{|c|}{0.05} & \multicolumn{2}{|c|}{0.1} \\
\hline & Recovery (\%) & RSD (\%) & Recovery (\%) & RSD (\%) & Recovery (\%) & RSD (\%) & Recovery (\%) & RSD (\%) \\
\hline \multirow[t]{3}{*}{ Rice } & 97.8 & & 87.5 & & 96.7 & & 94.7 & \\
\hline & 98.8 & 1.3 & 86.6 & 1.2 & 96.1 & 2.3 & 89.8 & 3.8 \\
\hline & 96.6 & & 85.9 & & 93.2 & & 92.3 & \\
\hline
\end{tabular}


This work has been funded by the eleventh 5-year Key Technologies RQD Program of China (Project No. 2006BAD08B07-2-8) and Natural Science Foundation of China (No. 20807052). The authors also thank Dr. J.-M. Lin of Tsinghua University for giving kind advices and support during the research.

The authors have declared no conflict of interest.

\section{References}

[1] Reiss, J., Food Cosmet. Toxicol. 1977, 15, 303-307.

[2] Nguyen, M. T., Tozlovanu, M., Tran, T. L., Pfohl-Leszkowicz, A., Food Chem. 2007, 105, 42-47.

[3] Xu, B., Jia, X., Gu, L., Sung, C., Food Control 2006, 17, 271-285.

[4] Baggiani, C., Anfossi, L., Giovannol, C., Anal. Chim. Acta 2007, 591, 29-39.

[5] Turner, N. W., Piletska, E. V., Karim, K., Whitcombe, M., Malecha, M., Magan, N., Baggiani, C., Piletsky, S. A., Biosens. Bioelectron. 2004, 20, 1060-1067.
[6] Li, X., Husson, S. M., Malecha, M., Magan, N., Baggiani, C., Piletsky, S. A., Biosens. Bioelectron. 2006, 22, 336-348.

[7] Yu, J. C. C., Lai, E. P. C., Food Chem. 2007, 105, 301-310.

[8] Jodlbauer, J., Maier, N. M., Lindner, W., J. Chromatogr. A 2002, 945, 45-63.

[9] Urraca, J. L., Marazuela, M. D., Merino, E. R., Orellana, G., Moreno-Bond, M. C., J. Chromatogr. A 2006, 1116, 127-134.

[10] Wang, X.-J., Xu, Z.-L., Feng, J.-L., Bing, N.-C., Yang, Z.-G., J. Membr. Sci. 2008, 313, 97-105.

[11] Kim, H., Guiocho, G., J. Chromatogr. A 2005, 1097, 84-97.

[12] Zhang, H., Song, T., Zhang, W., Hua, W., Pan, C., Bioorg. Med. Chem. 2007, 18, 6089-6095.

[13] Lele, B. S., Kulkarni, M. G., Mashelkar, R. A., Reactive Funct. Polymers 1999, 39, 37-52.

[14] Martin, P., Wilson, I. D., Jones, G. R., J. Chromatogr. A 2000, 889, 143-147. 